\title{
Article
}

\section{Development of the post-2015 framework for disaster risk reduction}

\section{Article Type: Editorial}

\section{From: International Journal of Disaster Resilience in the Built Environment, Volume 5, Issue 4}

Many readers of IJDRBE will agree that the Hyogo Framework for Action (HFA) has provided important guidance to reduce disaster risk and strengthen cooperation across stakeholders at multiple levels. However, its implementation has also highlighted important gaps in the formulation of goals and priorities for actions. For example, while priorities 1,2,3 and 5 were often deemed to be directly actionable and specific, priority 4 has proven to be challenging. As a result, a post-2015 framework for disaster risk reduction is now being developed to update and reorder the strategic goals and priorities, giving appropriate visibility to all levels and placing greater emphasis on stakeholders and their role in advancing the priorities.

Since the adoption of the HFA, countries in all regions have been reporting steady progress in strengthening their institutional, legislative and policy frameworks. Many have suggested that this has contributed to decreasing mortality risk, especially from floods and tropical storms. Progress has also been made in risk assessment, education, research and public awareness, and many countries have been increasing their investments in risk reduction, as well as developing risk-transfer mechanisms. Such reports suggest that the HFA has been an important instrument in raising institutional awareness and understanding, while also instilling political will.

Despite this positive evaluation, biennial reports of countries on the HFA implementation indicate that exposure of people and assets in all countries have been increasing faster than vulnerability has been decreasing. This has resulted in new risk and increasing disasters losses, with significant socioeconomic impact in the short-, medium- and long terms, especially at the local and community level.

The Co-Chair's pre-zero draft of the post-2015 framework for disaster risk reduction notes that:

There are risk factors which have not received sufficient attention and indeed constitute underlying risk drivers. Factors such as unequal economic development, poorly managed urban development and ecosystems, poverty and inequality, weak participatory governance, weak enforcement, insufficient local capacities, inadequate and inappropriate policies and resources, conflicts, and climate change and variability compound disaster risk and hence the levels of disaster loss.

Despite progress, there is evidently much work ahead and these factors can certainly inform future research agendas.

Perhaps of most relevance to the readers of IJDRBE, the pre-zero draft also identifies an important role for academia and research, who are encouraged to:

[...] focus on the evolving nature of risk and scenarios in the medium and long terms; increase research for local application and support to local communities and authorities' action; and support the interface policy-science for effective decision making.

This judgment aligns well with IJDRBE's remit. Since launching in 2010, IJDRBE has been reporting research that assists capacity-building for reconstruction, renewal and development of sustainable infrastructure, supports proactive and fruitful collaborations and networking among various stakeholders and helps develop appropriate policy development and plans for implementation. 
In doing so, the journal has been working towards it key objectives: developing the skills and knowledge of built environment researchers and professions working in disaster prone areas, so that they may strengthen their capacity in strategic and practical aspects of disaster prevention, mitigation, response and reconstruction; provide a unique forum for novel enquiries into the development and application of new and emerging practices as a source of innovation to challenge current practices; promote the exchange of ideas between researchers, educators, practitioners and policy makers; and influence disaster prevention, mitigation, response and reconstruction policies and practices.

Authors are directly encouraged to address and highlight the practical and social implications of their research during the paper submission process. While optional, authors are requested to identify any practical or social implications: What outcomes and implications for practice, applications and consequences are identified? Not all papers will have practical implications but most will. What changes to practice should be made as a result of this research/paper? What will be the impact on society of this research? How will it influence public attitudes? How will it influence (corporate) social responsibility or environmental issues? How could it inform public or industry policy? How might it affect quality of life?

Prior to introducing this issue's content, it is also worth noting that in Volume 5 of IJDRBE, the journal has increased from three to four issues. This reflects the growing importance of the topic, an increasing readership and the continued support of our publisher, Emerald.

Opening this final and fourth issue of volume five, Nakanishi, Black and Matsuo examine the transportation organizational service provision and travel behavioural responses after the March 2011 disaster in North East Japan. Their study reveals that transportation demand changes dramatically in the emergency phase, while in the re-building phase, an efficient and effective provision of a transportation service is required.

In the second paper, Cai, Rahman, Su and Zhang present a study of timely evacuation that considers the dynamic traffic demand and congestions under dynamic hazard conditions. University campuses face a unique challenge in developing effective emergency evacuation plans due to their complex site configurations, high-dense buildings and dynamic spatial-temporal distribution of population. They conclude that their newly created framework and its implementation could assist emergency evacuation planning for university campuses.

Korstanje explores semantically the connection between national being and five indicators: beautiness, coaction, competence, stratification and monetary attachment, and considers how nationalism evolves following a disaster.

In the fourth article, Jordan, Javernick-Will and Amadei examine why communities facing the same disaster recover differentially and determine pathways to successful disaster recovery in the research setting of New Orleans neighbourhoods affected by Hurricane Katrina. Their results show that there are multiple pathways combining pre-disaster community factors and post-disaster actions that led to recovery, as measured by population return.

Shieh, Habibi, Torabi and Masoumi by using indexes such as access to medical centres, street inclusion, building and population density, land-use and building quality, study the earthquake vulnerability of street networks in an area of Tehran. They calculate vulnerability through overlaying maps and data in combination with the inversion hierarchical weight process method and geographic information systems. 
In the final research article, Mandal provides a review of supply chain resilience and identifies research gaps in risk management, supply chain design, sourcing strategies, green practices, sustainable competitive advantage, supply chain security, supply chain performance and supply chain resilience.

Korstanje contributes a review of Individuación, precariedad, Inseguridad [individuation, precarity and insecurity] by Robert Castel, Gabriel Kessler, Denis Merklen and Numa Murard. The issue concludes with news of the 3rd World Conference on Disaster Risk Reduction, which will be held from 14 to 18 March 2015 in Sendai, Japan.

Richard Haigh and Dilanthi Amaratunga 